\title{
A desconstrução do conceito de DeficiênCia Mental e a construção DO CONCEITO DE INCAPACIDADE INTELECTUAL: DE UMA PERSPETIVA ESTÁTICA A UMA PERSPETIVA DINÂMICA DA FUNCIONALIDADE
}

THE DECONSTRUCTION OF THE MENTAL RETARDATION CONCEPT AND THE CONSTRUCTION OF THE INTELLECTUAL DISABILITY CONCEPT: FROM A STATIC TO A DYNAMIC PERSPECTIVE OF FUNCTIONING

\author{
Manuela SANCHES-FERREIRA ${ }^{1}$ \\ Pedro LOPES-DOS-SANTOS ${ }^{2}$ \\ Miguel Augusto SANTOS 3
}

\begin{abstract}
RESUMO: as questôes em torno da deficiência, da incapacidade e da funcionalidade tornaram-se, nas últimas décadas, importante foco de discussão e de elaboração conceitual, com a produção de um corpo de saberes que confere legitimidade científica a uma visão mais positiva e dignificante das condiçōes de deficiência e de incapacidade. A premissa base dessa produção de conhecimento é a assunçấo da reciprocidade das relaçóes indivíduo/meio, enquanto unidade nuclear de análise, onde a incapacidade é encarada, não como característica intrínseca da pessoa, mas como o resultado do desajustamento entre as funcionalidades do indivíduo e as solicitaçóes dos cenários onde ele é chamado a participar. É nesta base que, neste artigo, nos propomos examinar as implicaçóes que tais posicionamentos tiveram na desconstruçáo do construto de deficiência mental (mental retardation) e subsequente mudança para a designação incapacidade intelectual (intellectual disability), pela American Association of Intellectual and Developmental Disabilities. Para atingir esse propósito discutiremos a evolução do conceito de deficiência mental, explorando o território conceitual que o instituiu e aquele que contribui para a sua desconstruçáo. Por último, a enunciação teórica deste artigo pretende contribuir para demonstrar a interdependência existente entre modos de pensamento e modos de ação, e que no caso da Educação Especial é corporizado na aceitação progressiva do paradigma da inclusão.
\end{abstract}

PALAVRAS-CHAVE: Educação Especial. Incapacidade Intelectual. Funcionalidade.

\begin{abstract}
Issues concerning impairment, disability and functionality have become, in recent decades, an important focus of discussion and conceptual elaboration, with the production of a body of knowledge that gives scientific legitimacy to a more positive and dignifying vision about the conditions of disability and impairment. The basic premise of this knowledge production is the assumption of reciprocity of the relations' individual/environment, as the basic unit of analysis, conceiving disability not as an intrinsic characteristic of the person, but as the result of the mismatch between the functionalities of the individual and the demands of the settings where persons are called to participate. Hence, in this article, we propose to examine the implications that such views had in the deconstruction of the construct of mental retardation and subsequent changes to the designation intellectual disability, by the American Association of Intellectual and Developmental Disabilities. To accomplish this purpose we discuss the evolution of the concept of mental retardation, exploring the conceptual territory that established it and the one that contributes to its deconstruction. Finally, the theoretical standpoint of this paper aims to demonstrate the interdependence between modes of thinking and modes of action that, in the case of Special Education, is embodied in the gradual acceptance of the inclusion paradigm.
\end{abstract}

KEYWORD: Special Education. Intellectual Disability. Functioning.

\footnotetext{
${ }^{1}$ Escola Superior de Educação do Instituto Politécnico do Porto. Unidade Técnico-Científica de Educação Especial. manuelaferreira@ese.ipp.pt

${ }^{2}$ Faculdade de Psicologia e de Ciências de Educação da Universidade do Porto. plsantos@fpce.up.pt

${ }^{3}$ Escola Superior de Educação do Instituto Politécnico do Porto. Unidade Técnico-Científica de Educação Especial. migsantos@ ese.ipp.pt
} 


\section{INTRODUÇÃO}

A evolução do conhecimento em Educação Especial tem sido pautada pela consolidação progressiva de modelos conceituais mais dinâmicos e interativos no entendimento dos fenômenos da incapacidade e da funcionalidade, onde facilmente se reconhecem as influências provenientes dos paradigmas ecológico, comportamental e biopsicossocial.

Estes modelos mais dinâmicos têm sustentado uma nova narrativa na descrição da interação entre a pessoa e o meio, como na revolução de Copérnico - que desmente o modelo representacional do Sol a girar em torna terra, mostrando tratar-se da situação inversa, - também assistimos, no domínio do desenvolvimento, a uma nova representação à luz da qual a criança deixa de gravitar em torno de um meio estático e imóvel, para passar a ser o meio dinâmico a gravitar em torno da criança. Contudo, a metáfora do sistema solar revelar-se-á insuficiente para retratar a complexidade das relaçóes de interdependência e de transacionalidade entre a criança e o meio, pois como referem Bijou e Baer (1978),

[...] Não podemos analisar a criança sem referência ao contexto e não é possível analisar o meio sem referência à criança. Os dois termos formam uma unidade inseparável e consistente com um conjunto inter-relacionado de variáveis que organizam um verdadeiro campo interativo. (p. 28)

Esta dinâmica que coloca, incontornavelmente, a reciprocidade das relaçóes indivíduo/meio enquanto unidade nuclear de análise, leva-nos a encarar a incapacidade, não como característica intrínseca da pessoa, mas como o resultado do desajustamento entre as funcionalidades do indivíduo e as solicitaçóes dos cenários onde ele é chamado a participar (OMS, 2001; LUCKASSON et al., 2002; SCHALOCK et al., 2010).

Assumimos - tal como Sanches-Ferreira (2007) - que, do ponto de vista do estado da arte, vivemos um momento de congruência conceitual, ética e pragmática à luz do qual o movimento inclusivo se consagra como nova gramática de estruturaçáo da realidade, engendrando uma gestalt irredutível à soma das confluências que a sustentam.

É, então, este o racional onde inscrevemos as propostas de desconstrução do construto de deficiência mental $l^{4}$ e de construção do construto de incapacidade intelectual constituindo o leitmotiv deste artigo.

\section{O Significado de DeficiêNCIA MENTAL}

Perguntar o que é a deficiência mental poderá gerar sentimentos de estranheza ou até de um certo desconforto, pois, conforme Greenspan (1999) aduz, se há certeza que, normalmente, tomamos como inquestionável é a de que existem pessoas inteligentes e outras que nem por isso. Todavia, apesar dessa aparente evidência, têm surgido vozes, desde há vários anos, a assumir que a deficiência mental reflete mais uma construção discursiva do que propriamente um conceito apoiado na observação cuidada e crítica dos fatos. Recordamos, a propósito, a posição de Sarason (1985) para quem a noção de deficiência mental não deve ser compreendida

\footnotetext{
${ }^{4}$ Neste artigo utilizaremos a designaçáo de Deficiência Mental como traduçáo de Mental Retardation, por corresponder à que tem sido utilizada em Portugal (veja-se a designação de algumas das principais instituiçôes que trabalham nesta área como por exemplo a Associação Portuguesa de Pais e Amigos do Cidadão Deficiente Mental e a Humanitas - Federaçáo Portuguesa para Deficiência Mental).
} 
como coisa ou característica de certos indivíduos, mas antes como o resultado de uma criação social coerente com os valores e ideologias dominantes de dada circunstância histórica onde as funções de diagnóstico e de prognóstico parecem representar operaçóes desejáveis e necessárias.

\section{A DeficiênCIA Mental Como CONSTRUTo e COMO NOME}

Ao considerarmos deficiência mental como um construto, estamos a atribuir-lhe o estatuto de ideia abstrata geral, dependente da organização das partes, baseada em fenômenos observados e suportada por uma determinada teoria.

Tal posição é também assumida por Luckasson et al. (1992) quando aduz que designar esse construto como deficiência mental, é reconhecê-lo como entidade singular, passível de ser diferenciada de outras entidades e, desse modo, favorecer a comunicação.

Nesta base, desconstruir o construto de deficiência mental passa, antes de tudo, pela análise das suas partes constituintes que, se atentarmos nas diferentes definiçóes constitutivas produzidas durante o último século, se organizam em torno de dois conceitos chave-inteligência e comportamento adaptativo - e cuja validade é fundamentada em teorias. Assim, desconstruir o construto implica:

1. Esclarecer a validade dos conceitos sobre os quais repousam as definiçóes;

2. Determinar se, realmente, nos deparamos perante uma entidade singular.

\section{INTELIGÊNCIA E $Q I$}

Em termos gerais e práticos, a inteligência tem sido, predominantemente, representada por um número - o Quociente de Inteligência (QI). Este número quantifica o desempenho do indivíduo face a uma série de problemas e em referência aos resultados da distribuição amostral obtida junto de uma quantidade significativa de respondentes que refletem as características da população à qual o indivíduo pertence. A distribuição em causa obedece, pois, aos parâmetros da curva normal obtida através de procedimentos de padronização dos resultados que situam no valor de 100 a sua nota média. A escolha deste valor não é inteiramente arbitrária, uma vez que pretende manter relaçóes de homologia com a escala criada através da aplicação da célebre fórmula de Stern, utilizada para obter medidas gerais da inteligência a partir da idade mental.

Uma abordagem rápida à história do conceito de inteligência - introduzido na literatura científica por Galton em finais do século XIX - mostra como, desde o seu início, a inteligência foi concebida como um traço ou disposição estável (1883).

Galton, tido como um dos primeiros eugenistas, sustentava que a inteligência era passível de ser medida e estudada cientificamente, tendo passado, de fato, grande parte da sua vida a tentar demonstrar a origem inata da inteligência. A pregnância desses primeiros estudos levou a que as teorias de natureza inatista constituíssem, durante muito tempo, o olhar dominante na ciência. A importância atribuída aos fatores endógenos funcionou como premissa orientadora da investigação que atribuiu especial relevo às capacidades ou incapacidades dos indivíduos na abordagem das aprendizagens acadêmicas. 
A procura da medida da inteligência levou à expansão da psicometria e à tentativa de encontrar modos de melhor apreender o conceito, durante toda a primeira metade do século XX. Esta perspectiva concebia a inteligência como a expressão de uma capacidade geral (fator g), englobando todos os processos cognitivos significativos, pouco permeável à idade ou à experiência e suscetível de ser avaliada numa única dimensão métrica. Esta ideia de um fator geral, que satura os domínios de competência específica (fatores s) encontra nos estudos de Spearman (1927) o seu expoente máximo. Em 1934, Burt, Jones e Miller (cit in GLASER, 1977) definiam inteligência como uma qualidade

[...] herdada, ou pelo menos inata, não dependente do ensino ou treino. É intelectual, não emocional ou moral, e permanece não afetável pelo esforço ou zelo; é geral, não específica, isto é, não está limitada a um tipo particular de trabalho, mas entra em tudo que nós fazemos, dizemos e pensamos. (p.49)

É neste pressuposto de inteligência, como algo de eminentemente intrínseco e estático (logo estável) que se baseiam os testes de inteligência e surge a primeira premissa do construto da deficiência mental: funcionamento intelectual abaixo da média. Ora, a fim de podermos reconhecer a inteligência como qualidade intrínseca, estável e imutável, ela terá de se manter invariável para um mesmo indivíduo ao longo do tempo e não ser suscetível de influência externa.

O melhor terreno para avaliar o caráter intrínseco da inteligência é fornecido pelo caso de certas síndromes relacionados com anomalias genéticas. Autores como Clunies-Ross (1990) ou Kliewer e Biklen (1996) referem que, por exemplo, os indivíduos com Síndrome de Down - condição resultante de mutação genética no cromossoma 21 - eram, durante as primeiras décadas do século XX, tipicamente classificados na categoria de deficiência mental profunda (QI menor que 20). Por volta de 1950, o seu QI médio situava-os no grupo da deficiência mental severa (QI entre 20 e 34). Na década de 1960, 10\% eram considerados educáveis, isto é, classificáveis no grupo da deficiência mental moderada (QI entre 35 e 49). Por sua vez, os estudos efetuados a partir dos anos de 1970 indicam que 20 a 50\% surgiam, já, em posição moderadamente abaixo da média, sendo uma percentagem pequena incluída nos limites da média.

Procedemos a esta menção para retirarmos a mesma conclusão extraída pelos autores acabados de referenciar: como a natureza e características da síndrome não mudaram, só podemos atribuir a evolução das médias do QI a fatores externos, ou dito de outro modo, à qualidade e aos esforços habilitativos do meio. A mesma situação é extensiva a outras síndromes de etiologia genética responsáveis por grande parte das situações de deficiência mental como sejam as síndromes de Prader-Willi, de Dandy-Walker, de Williams ou de Bardet-Biedel que foram associados a deficiência mental severa ou profunda e agora são encarados como podendo ter casos nos níveis mais elevados da deficiência mental ligeira ou, até, na zona do QI médio (e.g., GREENSPAN, 1999; MARTENS; WILSON; REUTENS, 2008; SASAKI-ADAMS et al., 2008; WHITTINGTON; HOLLAND; WEBB, 2009). Assim, o caráter inato e imutável da inteligência resulta questionável, pois os efeitos associados às síndromes genéticas seriam a melhor comprovação de tal atributo. 
Um dos problemas deste entendimento de QI tem a ver com a associação rapidamente estabelecida entre QI e prognóstico de capacidade. Foi, precisamente, esta associação que orientou, predominantemente, todas as práticas educativas e sociais até aos anos de 1960, legitimando políticas de institucionalização ou de educação segregada. De fato, olhar o indivíduo através dos seus déficits foi subsidiário da conclusão de que o seu funcionamento intelectual se diferenciava não só quantitativamente, mas também qualitativamente. É, aliás, esta última característica que justifica o grande esforço colocado no treino e não na educação dos alunos cuja inteligência correspondia aos níveis de deficiência mental profunda, severa e, não raramente moderada.

\section{O COMPORTAMENTO ADAPTATIVO}

As críticas à classificação de crianças através de testes de inteligência foram crescendo de tom, quer pelos estudos em torno do conceito de inteligência, quer pelas sucessivas mudanças nos critérios. É neste panorama que o conceito de comportamento adaptativo aparece de forma explícita na definição de Heber (1961) sob a égide da American Association on Mental Retardation (AAMR) e, a partir da qual, a investigaçáo procura encontrar metodologias que permitam avaliar o comportamento adaptativo.

Surgem as primeiras escalas construídas para o efeito, como, por exemplo, a de Vineland Social Maturity Scale (DOLL, 1953) mais tarde transformada na Vineland Adaptative Behavior Scale (SPARROW; BALLA; CICCHETTI, 1984). Esta solução não colheu grande consenso, pois, para além destes instrumentos terem sido objeto das mesmas críticas feitas aos testes de QI (KAMPHAUS, 1987), acresce, ainda, o fato de serem preenchidos através de rating scales baseadas em entrevistas. Assim, a introdução deste critério dual, na prática, não funcionou e o diagnóstico continuou a basear-se no QI, ignorando, de fato, as componentes comportamentais.

A polêmica em torno da necessidade deste segundo critério de diagnóstico organizase em duas linhas de argumentação antagônicas, mas consensuais no que diz respeito à dificuldade de encontrar medidas adequadas do comportamento adaptativo (GREENSPAN, 2006). Uma linha defende a inutilidade desta medida, uma vez que o QI seria suficiente, pois pressupóe já, implicitamente, uma desordem de comportamento; no outro extremo refuta-se tal afirmação na medida em que não há evidência empírica nem teórica de que deficiência mental seja sinônimo de desordem global ao nível do comportamento adaptativo. Isso mesmo acaba por transparecer na definição de 1992 da AAMR, onde comportamento adaptativo passa a ser designado por competência adaptativa.

Partimos, então, para a análise de deficiência mental como entidade singular com a descrença de Hobbs (1975) nos sistemas de classificação, por serem inadequados, por obscurecem a individualidade da criança, e pelas categorias de deficiência terem um valor limitado em educação. 


\section{DEFICIÊNCIA MENTAL: ENTIDADE SINGULAR}

O reconhecimento da deficiência mental enquanto entidade nosográfica suscetível de diagnóstico pressupóe que falemos de uma categoria correspondente a uma entidade singular. Segundo Datta e Farradane (1974), classificar corresponde a uma organização dos nossos conceitos ou construtos mentais acerca das entidades e elementos externos. De fato, são vários os domínios do conhecimento, cujo objetivo dos sistemas de classificação é contribuir para diferenciar, ordenar e agrupar de forma homogênea. Mas a possibilidade de classificarmos assenta na existência de uma homogeneidade das qualidades inerentes às entidades que são objeto de classificação. No caso da deficiência mental, a qualidade inerente por excelência, que assenta a classificação, é a inteligência medida através dos testes de inteligência. Sendo assim, trata-se de ilustrar se os testes de QI são apropriados como instrumentos de diagnóstico e, subsequentemente, capazes de capturar a essência da inteligência.

Um teste de inteligência reúne todo um conjunto de tarefas ou problemas cuja resolução pretende apelar a competências consideradas representativas da capacidade intelectual. Para isso os testes terão de possuir consistência, o que não parece demonstrado na investigação.

Contudo, já em 1989, Ysseldyke referia não haver, até à altura, nenhum procedimento psicométrico válido para diferenciar crianças em categorias, nem tampouco evidência que suportasse a noção de que alunos de categorias diferentes aprendiam de modo diferente. Baseava a sua afirmação num conjunto de estudos realizados por ele e seus colaboradores cujas conclusóes apontavam para a circunstância de mais de $80 \%$ dos alunos normais poderem ser classificados como tendo Dificuldades de Aprendizagem por uma ou mais das definiçóes aceites na altura, e que só $75 \%$ dos alunos com Baixo Rendimento Acadêmico cumpriam os critérios da classificação de Dificuldades de Aprendizagem. Estas constataçóes levaram-no a concluir que o então sistema de classificação não cumpria os preceitos de validade, de abrangência, de consistência lógica, de utilidade e de acordo entre os observadores.

Também Martin (1995) ao elaborar uma sinopse das investigações sobre a temática da colocação educacional encontrou nos estudos publicados - entre 1965 e 1992 - 68 rótulos diferentes para identificar os grupos de alunos.

Prova indireta desta dificuldade de apreender a categoria de deficiência mental tem sido o esforço da AAMR de adequar a sua definição a uma realidade que parece escapar cada vez mais. Estamos a referirmo-nos à volubilidade relativa aos desvios padróes na base dos quais se assume o diagnóstico de deficiência mental. Assim, e referindo apenas os momentos mais significativos dessa evolução temos em 1961, na definição de Heber, o nível Bordeline respeitante ao QI entre 65 e 83; leve (50-66); moderado (33-49); severo (16-32) e, por último, profundo correspondente a um QI abaixo de 16.

Em 1973, Grossman elimina o grau de bordeline, passando a referir como funcionamento intelectual subnormal aquele que se situa entre 70 e 85 , o que eliminou do diagnóstico um número significativo de indivíduos. Em 1977, Grossman desce o limite a partir do qual se é classificado com deficiência mental para 70-75. Face a esta arbitrariedade, não admira que, inúmeras vezes, a deficiência mental seja referida na literatura como classificação administrativa, (GREENSPAN, 2006), pois serve mais questóes de elegibilidade do que uma 
real diferenciação entre indivíduos (um desvio padrão a mais significa a diminuição de $10 \%$ para 3\% de população classificada como tendo deficiência mental).

Parece, então, legítimo concluir que deficiência mental não é uma entidade singular, já que dificilmente consegue ser isolada de forma inequívoca.Esta realidade é há muito explicitada na literatura científica e até com humor. Por exemplo Mercer (1973), entre muitos, ironizou referindo as crianças em idade escolar como "crianças com deficiência mental por seis horas", pois são-no na sala de aula, mas não nos outros contextos (família ou comunidade). Podemos, até, recuar mais algumas décadas e relembrar alguns dos posicionamentos teóricos de Zazzo datados de 1946 (a;b), que já contemplava a ideia de que $o$ débil mental ligeiro era filho do sistema público obrigatório.

\section{A Mudança de Paradigma}

Nas ciências humanas não é fácil situarmos numa data precisa as mudanças de paradigma, isto é, quando uma corrente de pensamento deixa de ser predominante na explicação dos fenômenos para dar lugar a uma outra. Podemos, isso sim, caracterizar esse processo como uma confluência de contributos e variáveis que se vão articulando entre si na descrição e explicação da deficiência e da incapacidade.

Destacamos, nessa confluência, os contributos das teorias da Aprendizagem Social, das Teorias Comportamentais e das Teorias Ecológicas e Transacionais, todas a colocarem os contextos e as interaçóes indivíduo-meio, na ordem do dia. De um modo simples, poderemos afirmar que a crença unificadora de todas estas concepçóes é a "modificabilidade do indivíduo, através da manipulação das contingências do meio ou das estruturas interativas subsistentes entre os vários cenários de participação imediata da criança” (SANCHES-FERREIRA, 2007, p.39).

Recuando um pouco mais no tempo na procura de contributos para essa confluência conceitual, é de referir, ainda, a antropologia e a etologia - com as suas metodologias observacionais - e o construtivismo social - com o conceito de realidade subjetiva - para a consolidação da mudança que vimos a enunciar.

De fato, é este "arsenal" conceptual que permitiu construir outra gramática, outra gestalt na base da qual ganhou solidez científica e se instituiu o movimento inclusivo.

Desta evolução científico-metodológica resultou a substituição da perspetiva biomédica na abordagem da deficiência - o déficit como qualidade ôntica do sujeito - por uma outra onde a relação entre o déficit e o contexto foi alterada, na procura de melhor descrever o funcionamento dos indivíduos com ênfase particular na consideração dos seus desempenhos de participação.

De um modo simplista, poderemos dizer que esses contributos se reorganizaram em duas grandes linhas unificadoras do pensamento e da investigação: o Modelo Social e o Modelo Biopsicossocial.

O Modelo Social salientou-se pelas propostas cuja premissa fundamental é a rejeição da incapacidade (traduzido do termo inglês disability) como uma característica interna da pessoa e a defesa de que esta resulta de uma construção social cujo objetivo tem sido discriminar e excluir os indivíduos (SIMEONSSON, 2006). Consentâneo com tal princípio, o esforço deste 
movimento tem sido colocado na mudança da legislação, demonstrando a importância que as barreiras físicas e psicossociais existentes assumem na segregação (SANCHES-FERREIRA et al., 2012; SILVEIRA-MAIA et al., 2012).

O Modelo Biopsicossocial agrupa as linhas de investigação cuja primeira preocupação é a conceptualização e medida da incapacidade (SIMEONSSON, 2006). A incapacidade, ao ser definida como um grande chapéu que abriga os conceitos de deficiência, limitação de atividade e restrição na participação (OMS, 2001) torna necessário uma medida rigorosa dos fatores do ambiente (barreiras e facilitadores) e das variáveis do indivíduo (capacidade e desempenho) implicados na sua funcionalidade.

O caminho ficou, então, aberto para que a investigação se debruçasse sobre a descrição dos processos, elegendo a pessoa-meio como unidade de análise, fugindo de

[...] modelos [...] de pouca utilidade para a solução dos problemas reais uma vez que na sua maioria assentavam em generalizaçóes de resultados obtidos em estudos e investigação efetuados ou em contextos laboratoriais ou em contextos afastados da prática educativa. (BAIRRÁO, 1995, p.13).

\section{FunCIONALIDADE E INCAPACIDADE}

Os modelos oferecem todo um conjunto de definiçóes e princípios que veiculam visóes específicas de olhar a realidade. De fato, as escolhas feitas para se descreverem os fenômenos determinam a maneira como estes são abordados e problematizados.

Tal constatação é aplicável à análise de incapacidade, pois enquanto ocorrência tem sido ao longo dos tempos retratada segundo diferentes pontos de vista. Essas perspectivas formataram linhas distintas de inquirição científica que se refletem ao nível das formulações teóricas e das pragmáticas de intervenção, bem como ao nível dos conceitos e das terminologias associadas aos diferentes modelos de incapacidade como é representado no Quadro 1. Como se pode apreciar, até à década de noventa do século passado, o pensamento dominante era o de que a presença de patologia com alguma gravidade constituía motivo suficiente para o indivíduo apresentar uma incapacidade em qualquer contexto. Por outras palavras, certas condiçóes patológicas - doença, anomalia genética ou de outra ordem - inscreviam-se numa linha de progressão que levava a uma deficiência - alteração significativa ao nível das estruturas ou funçôes de um sistema específico do corpo - que produzia limitaçóes funcionais - dificuldades na realização de atividades - cuja consequência era a incapacidade - restrições na vida do dia a dia. Efetivamente, é em 1997 que o Institute of Medicine (IOM) coloca os fatores ambientais e a qualidade de vida como variáveis neste processo, atribuindo-lhes, contudo, uma tênue importância na funcionalidade. 
Quadro 1- Evolução dos conceitos e terminologias usadas nos diferentes modelos de incapacidade (adaptado de WHITENECK, 2006).

\begin{tabular}{|l|l|l|l|l|l|}
\hline Modelo/ ano & Origem & Órgáos corpo & Nível individual & Nível Social & Outros domínios \\
\hline Nagi, 1976 & Patologia & Deficiência & Limitação funcional & Incapacidade & \\
\hline OMS, 1980 & Patologia & Deficiência & Incapacidade & Desvantagem & \\
\hline IOM, 1991 & Patologia & Deficiência & Limitação funcional & Incapacidade & \\
\hline NCMRR', 1992 & Patologia & Deficiência & Limitação funcional & Incapacidade & $\begin{array}{l}\text { Limitaçóes da vida } \\
\text { social }\end{array}$ \\
\hline IOM, 1997 & Patologia & Deficiência & Limitação funcional & Incapacidade & $\begin{array}{l}\text { Fatores ambientais, } \\
\text { qualidade de vida }\end{array}$ \\
\hline $\begin{array}{l}\text { OMS, } \\
\mathbf{2 0 0 1 , 2 0 0 7}\end{array}$ & $\begin{array}{l}\text { Condição } \\
\text { de saúde }\end{array}$ & $\begin{array}{l}\text { Estrutura e fun- } \\
\text { ção do corpo }\end{array}$ & Atividade & Participação & $\begin{array}{l}\text { Fatores ambientais, } \\
\text { fatores pessoais }\end{array}$ \\
\hline
\end{tabular}

${ }^{1}$ National Center for Medical Rehabilitation Research

Realmente, é o modelo da OMS $(2001,2007)$ que marca a ruptura com as anteriores concepçóes, dando um passo significativo na procura da explicitação desta dinâmica e na busca de uma linguagem cada vez mais neutra para descrever a realidade.

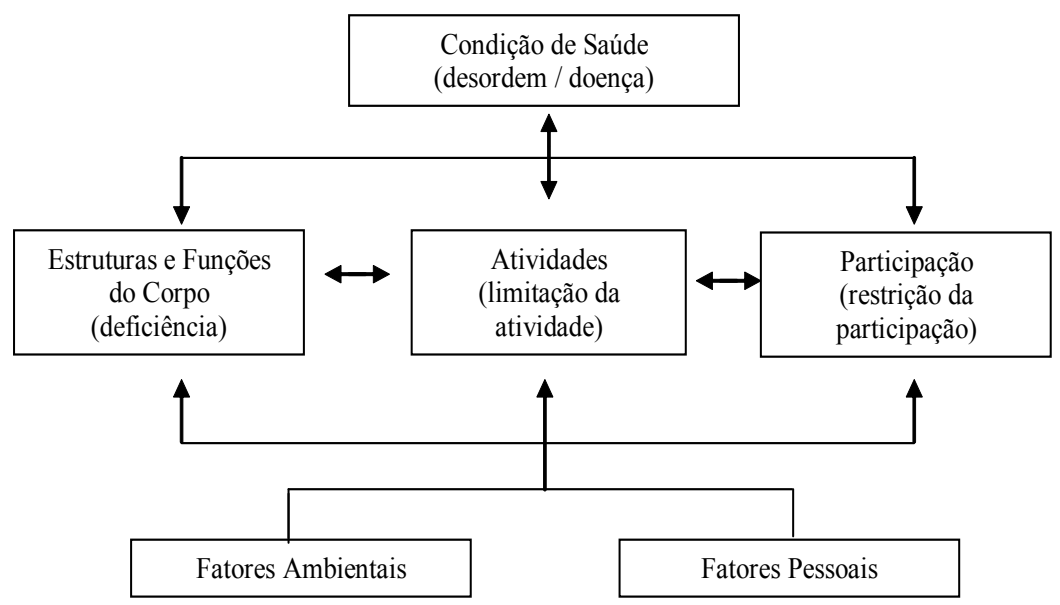

Figura 1 - Modelo de Incapacidade da Classificação Internacional da Funcionalidade (CIF) adaptado de Simeonsson e Lollar (2006).

A Figura 1 é uma representação do modelo de incapacidade, isto é, das relaçôes estabelecidas entre as condiçôes de saúde de um indivíduo e os fatores pessoais e do ambiente que podem afetar ou facilitar essa funcionalidade. Um olhar rápido permite reconhecer a substituição dos conceitos de patologia pelo de condição de saúde, de deficiência (Impairment) por funçóes e estruturas do corpo e da noção de limitaçóes funcionais por atividade. De igual 
modo, incapacidade (Disability) é tratada em termos de participação com os fatores ambientais a marcarem decisivamente este processo. A participação deixa de depender apenas das estruturas e funçóes do corpo, pois os fatores do meio são fundamentais.

Ao incluir os fatores físicos, sociais e do meio a interagirem com as condições de saúde, o modelo da OMS admite a importância potencial da manipulação do ambiente ao nível das atitudes, das condiçóes físicas, das políticas legislativas e sociais. Por outras palavras, propóe uma responsabilidade social, no sentido de uma verdadeira equidade.

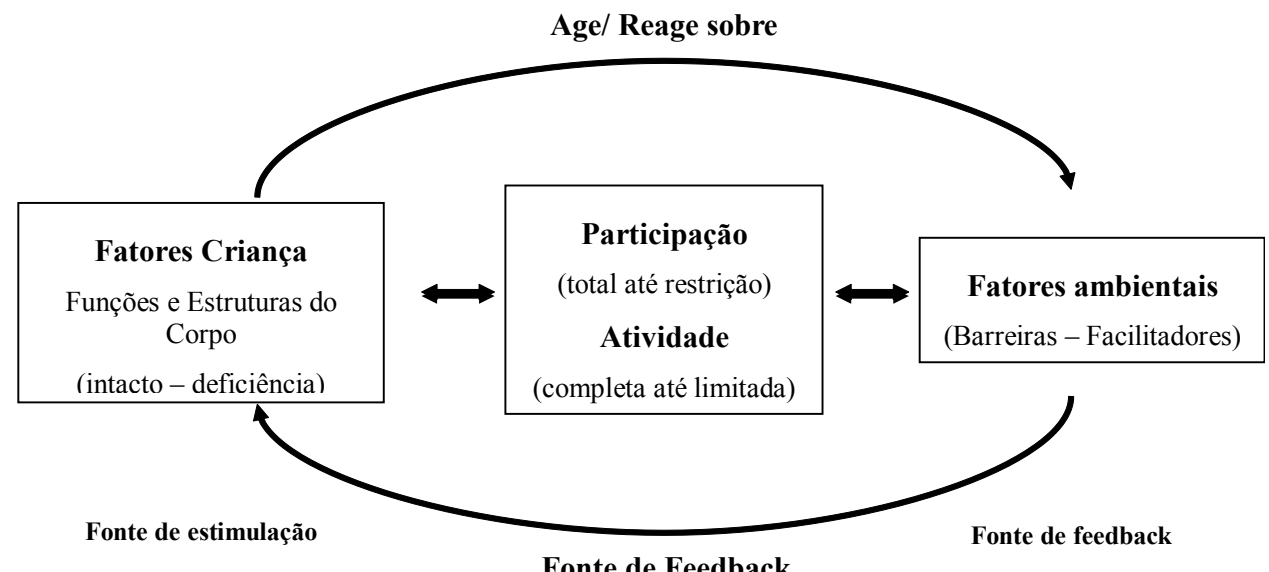

Figura 2 - Modelo da interação criança-ambiente da Classificação Internacional de Funcionalidade criança e jovem (CIF-CJ) adaptado de Simeonsson e Lollar (2006).

Por conseguinte, como se pode apreciar na representação avançada na Figura 2 o processo de desenvolvimento e o processo de incapacitação funcionam como sistemas abertos e paralelos, dependente da retroalimentação do ambiente. Uma grande virtude desta proposta é, então, a atribuição ao ambiente do peso essencial na equação que torna possível a expressão capacidades iguais não significam funcionalidades iguais. De fato, a participação de uma pessoa com deficiência tem uma relação direta com os facilitadores e as barreiras que o ambiente lhe coloca (e.g. SILVEIRA-MAIA et al., 2012).

Deste modo, a Incapacidade passa a ser entendida num contínuo de situaçóes provocadas pelas relaçóes estabelecidas entre as diferentes variáveis envolvidas.

\section{A definiçáo de DeficiênCIa Mental da AAMr e o Modelo de Suporte}

A adoção dos modelos funcionais na explicação do funcionamento humano está patente nas definiçóes que a então American Association on Mental Retardation produziu em 1992 e, posteriormente, em 2002 ao introduzir mudanças significativas na definição operacional de deficiência mental. 
De fato, o sistema de definição e de classificação na proposta de 1992 da AAMR é como que um olhar ao espelho caracterizado pela eliminação dos quatro níveis de deficiência mental - leve, moderado, severo e profundo - e pela identificação de quatro níveis de intensidade de suporte necessário à funcionalidade do indivíduo, tendo como norma os pares da sua idade. Ora, nâo faz sentido, a partir de entâo, centrar a avaliação na pessoa, mas sim, na participação, isto é, na interação entre o indivíduo e o meio.

Dos pressupostos de avaliação, com vista a um diagnóstico de deficiência mental, fazem parte as seguintes cinco assunçóes (LUCKASSON et al., 2002, p.1):

1. A limitação no funcionamento presente deve ser considerada dentro do contexto do envolvimento na comunidade, típico para os pares ou indivíduos da mesma idade;

2. Uma avaliação válida é aquela que considera a diversidade cultural e linguística bem como as diferenças na comunicação e nos fatores sensoriais, motores e comportamentais;

3. No indivíduo as limitações coexistem com as forças;

4. Um propósito importante quando se descrevem limitações é o desenvolvimento de um perfil de necessidades de suporte;

5. Com suportes apropriados e individualizados durante um período adequado, o funcionamento no quotidiano da pessoa com deficiência mental geralmente aumenta.

Nível de suporte é, então, aquele que um indivíduo precisa para melhorar a sua funcionalidade podendo, ao longo da vida, necessitar, de diferentes tipos de suporte:

- Intermitente - suporte cuja base é o quanto necessário e caracteriza-se pela sua natureza episódica e pontual, podendo a intensidade ser diferente de momento para momento;

- Limitado - a intensidade é consistente ao longo do tempo, mas limitada em termos de duração;

- Extensivo - caracterizado por um envolvimento regular diário pelo menos em alguns contextos (e.g. casa, sala de aula...) e não limitado no tempo (isto é, suporte duradouro e suporte de longo prazo para as atividades da vida quotidiana);

- Invasivo - suporte caracterizado pela sua permanência, alta intensidade, fornecido transversalmente em vários contextos, podendo assumir a natureza de cuidados de sobrevivência; envolve, tipicamente, vários profissionais e é intrusivo.

A visão atrás enunciada inscreve-se numa avaliação funcional que terá de apreender a interação pessoa-meio, bem como a intensidade dos suportes necessários ao funcionamento.

Nesta perspectiva, e como num outro contexto um dos autores sublinha ${ }^{5}$ a funcionalidade pode ser considerada como uma equação onde o grau de funcionalidade é função das limitaçóes do indivíduo (denominador) e do apoio recebido (numerador). Assim, quanto maior o denominador - neste caso a limitação funcional - maior terá de ser o numerador, isto

${ }^{5}$ Ideia apresentada por Manuela Sanches-Ferreira nas Provas Públicas para Professora Coordenadora a 22 de Novembro de 2007 (trabalho nâo-publicado). 
é, o nível de suporte a fim de que a funcionalidade se mantenha a um determinado nível. É nesta oscilação que se joga o contínuo funcionalidade/ incapacidade.

Então, e face a esta mudança de paradigma, o modo como pensamos a deficiência mental alterou-se radicalmente. Luckasson et al. (2002) mostra eloquentemente esta alteração no modo de pensar e definir a deficiência mental ao definir como

um estado particular de funcionamento que existe baseado na relação entre as limitações funcionais da pessoa e os contextos em que esta existe ou funciona" e ao acrescentar que deficiência mental não é "algo que a pessoa tenha - olhos azuis, uma doença - nem algo que a pessoa seja - alta ou magra - nem uma desordem médica nem mental (p.10).

Ora, tal entendimento justifica a questão: ainda que se trate de significar a mesma população, poderia a designação permanecer inalterada?

\section{INCAPACIDADE INTELECTUAL}

Em 2007, a American Association on Mental Retardation, substitui formalmente a sua designação para American Association of Intellectual and Developmental Disability (AAIDD).

Ora, a mudança de nome, de uma instituição fundada em 1876, significa que o nome que damos às coisas ou fenômenos não é um assunto irrelevante.

Antes de tratarmos da substância do conceito de incapacidade intelectual atentemos na mera questão terminológica. Se considerarmos a tradução da palavra disability nas diversas versóes oficiais da CIF nas línguas de origem latina, verificamos que foram adotadas as seguintes designaçôes: handicap em francês, disabilità em italiano, dizabilității em romeno, discapacidad em espanhol e incapacidade em português. Parece, então, que excetuando a tradução para francês, todas as outras quatro recorrem a soluçáo idêntica, usando um prefixo de negação de uma habilidade ou capacidade.

Indo, agora, ao cerne do problema, segundo Luckasson e Reeve (2001), a atribuição de um nome deve cumprir cinco critérios:

1. Ser específico, isto é, referir-se a uma única entidade de modo a permitir a diferenciação de outra e estabelecer a comunicação;

2. Ser consistentemente usado por diferentes grupos de profissionais (psicólogos, professores, legisladores...);

3. Traduzir uma representação adequada do conhecimento atual e, simultaneamente, ser capaz de incorporar novos conhecimentos;

4. Ser suficientemente robusto na sua operacionalização para permitir vários propósitos, incluindo o diagnóstico, a classificação, e o planejamento de suportes;

5. Refletir uma componente essencial para nomear um grupo de pessoas comunicando valores importantes.

O termo incapacidade intelectual, desde logo conotado semanticamente com a função da inteligência, parece responder ao requisito da especificidade. A mesma asserção é, aliás, problemática relativamente ao uso da expressão deficiência mental já que o universo da palavra mental tem uma abrangência na qual não se incluem, apenas, as atividades ditas intelectuais, 
mas praticamente todas as outras dimensóes do psiquismo humano. Por outro lado, a referência a incapacidade intelectual coloca-nos num território conceptual heuristicamente mais rico, na medida em que, através da sua necessária ligação a processos de incapacitação estabelece interfaces profícuos com os modelos e paradigmas da Psicologia do Desenvolvimento Humano.

A incapacidade concebida enquanto manifestação de uma limitação do funcionamento do indivíduo dentro de um contexto social, leva-nos a olhar para a incapacidade intelectual como ocorrência que implica a unidade de análise indivíduo-meio com o consequente reconhecimento da importância dos sistemas de suporte no favorecimento da funcionalidade. Falarmos em incapacidade intelectual significa a assunção de terminologias associadas que comunicam valores importantes. É o caso de condição de saúde - e não doença ou patologia - estruturas e funçóes do corpo, atividade, participação - e náo deficiência. Como tal, o termo proposto parece responder de modo mais apropriado à exigência dos critérios acima enunciados.

\section{Conclusão}

Reynolds e Birch (1982) a propósito da evolução dos movimentos educativos, escreveram que a história da Educação Especial pode ser descrita como uma progressão sucessiva de encontro à inclusão. Um olhar rápido às denominações atribuídas à população, hoje designada com Incapacidade Intelectual, - idiotas, imbecis, débeis mentais, "handicap" mental, atrasados mentais, deficientes mentais -, mostra que a progressão do conhecimento favoreceu a procura não só de modelos mais compreensivos como de designaçóes cada vez mais neutras (SANCHES-FERREIRA, 2007). De fato, por detrás dessas designaçóes esteve o conceito de inteligência, que, segundo Sternberg (1995), "é um artefato cultural que esquecemos que foi por nós criado e, que mais tarde, tentamos explicar como se de um fenômeno natural se tratasse". Ora, o que os modelos funcionais e os modelos de suporte mostram é que o ambiente é a variável mais importante no funcionamento humano.

Assim, a designação de Incapacidade intelectual ao incorporar o termo incapacidade reenvia, forçosamente, para modelos dinâmicos e transacionais do desenvolvimento e, como tal, para uma procura constante do melhor ajustamento entre a pessoa e o ambiente. Será, seguramente, desse e nesse ajustamento, que será possível responder, como referem SilveiraMaia; Lopes-dos-Santos et al. (2012), às demandas éticas que postulam que todo o ser humano tem o direito a um papel social e a participar na comunidade onde está, naturalmente, inserido.

\section{REFERÊNCIAS}

BAIRRÃO, J. A perspectiva ecológica em psicologia da educação. Psicologia, v. 3, p. 7-30, 1995.

BIJOU, S. W.; BAER, D. M. Behavior analysis of child development. Engelwood Cliffs, NJ: PrenticeHall, 1978.

BIJOU, S. A functional analysis of retarded development. In: ELLIS, N. R. ELLIS (Ed.). International review of research in mental retardation (Vol. 1). New York: Academic Press, 1966. p. 1-19.

CLUNIES-ROSS, G. Intellectual disability - language and reading, in The Right to Read-Publishing for People with Reading Disabilities. Canberra, Australia: National Library of Australia, 1990. 
DATTA, S.; FARRADANE, J. E. L. A psychological basis for general classification. In: OTTAWA CONFERENCE ON THE BASIS OF THE CLASSIFICATION OF KNOWLEDGE, 1971, Ottawa. Proceedings...München: Verlag Documentation, 1974. p.319-331.

DOLL, E.A. The measurement of social competence: A manual for the Vineland Social Maturity Scale. US: Educational Test Bureau Educational Publishers, 1953.

GALTON, F. Inquiry into Human Faculty and its Development. London: MacMillan, 1883.

GLASER, R. Adaptive Education: Individual Diversity and Learning. New York: Holt, Rinehart and Winston, 1977.

GREENSPAN, S. What is meant by mental retardation?. International Review of Psychiatry, v.11, n. 1, p. 6-18, 1999.

GREENSPAN, S. Functional Concepts in Mental Retardation: Finding the Natural Essence of an Artificial Category. Exceptionality, vol. 14, no4, p. 205-224. 2006.

GROSSMAN, H. J. Manual on terminology in mental retardation. ed. Washington, DC: American Association on Mental Deficiency, 1973.

GROSSMAN, H. J. Manual on terminology in mental retardation. ed. Washington, DC: American Association on Mental Deficiency, 1977.

HEBER, R. A manual on terminology and classification on mental retardation. Revised ed. Washington, DC: American Association on Mental Retardation, 1961.

HOBBS, N. The Future of Children: Categories, Labels, and their Consequences. San Francisco: JosseyBass, 1975.

KAMPHAUS, R. W. Conceptual and Psychometric Issues in the Assessment of Adaptive Behavior. Journal of Special Education, v. 21, n. 1, p. 27-35, 1987.

KLIEWER, C.; BIKLEN, D. Labeling: Who wants to be called retarded? In: STAINBACK, W.; STAINBACK, S. (Eds.). Controversial issues confronting special education: Divergent perspectives. Boston: Allyn \& Bacon, 1996. p. 83-95.

LOPES-DOS-SANTOS, P. et al. The Supports Intensity Scale: Lessons from Portugal [abstract]. Journal of Intellectual Disability Research, v. 56, p. 798, 2012.

LUCKASSON, R.; REEVE, A. Naming, defining, and classifying in mental retardation. Mental Retardation, v. 39, p. 47-52, 2001.

LUCKASSON, R.. et al. Mental retardation: Definition, classification, and systems of supports. 10. ed. Washington DC: American Association on Mental Retardation, 2002.

LUCKASSON, R. et al.. Mental retardation: Definition, classification, and systems of supports. 9. ed. Washington DC: American Association on Mental Retardation, 1992.

MARTENS, M. A.; WILSON, S. J.; REUTENS, D. C. Research Review: Williams Syndrome: a critical review of the cognitive, behavioral, and neuroanatomical phenotype. Journal of Child Psychology and Psychiatry, v. 49, p. 576-608, 2008.

MARTIN, E. W. Case studies on inclusion: worst fears realized. The Journal of Special Education, v. 29, p. 192-199, 1995.

MERCER, J. Labeling the mentally retarded: Clinical and social system perspective on mental retardation. California: University of California Press, 1973. 
OMS. International Classification of Functioning, Disability and Health. Geneva: Organização Mundial de Saúde, 2001.

OMS. International Classification of Functioning, Disability and Health - version for children and youth. Geneva: Organização Mundial de Saúde, 2007.

REYNOLDS, M. C.; Birch, J. W. Teaching Exceptional Children in all America's Schools. 2. ed. Reston. VA.: Council for Exceptional Children, 1982.

RODRIGUES, D. A educação e a diferença. In: RODRIGUES, D. (Org.). Educação e Diferença: Valores e Práticas Para Uma Educaçáo Inclusiva. Porto: Porto Editora, 2001. p. 13-34.

SANCHES-FERREIRA, M. Educação Especial Educação Regular, Uma História de Separação. Porto: Afrontamento, 2007.

SANCHES-FERREIRA, M. et al. Portugal's special education law: implementing the ICF in policy and practice. Disability \& Rehabilitation, advance online publication, August 13, 2012.

SARASON, S. Psychology and mental retardation: Perspectives $m$ change. Austin, TX: PRO-ED, 1985.

SASAKI-ADAMS, D. et al. The Dandy-Walker variant: a case series of 24 pediatric patients and evaluation of associated anomalies, incidence of hydrocephalus, and developmental outcomes. Journal of Neurosurgical Pediatrics, v. 2, p. 194-199, 2008.

SPARROW, S. S.; BALLA, D.; CICCHETTI, D. Vineland Adaptive Behavior Scales. Circle Pines, MN: American Guidance Service, 1984.

SPEARMAN, C. The Abilities of Man. London: MacMillan, 1927.

SCHALOCK, R. L. et al. Intellectual Disability: Definition, Classification, and Systems of Supports. 11. ed. Washington D. C.: American Association of Intellectual and Developmental Disabilities, 2010.

SILVEIRA-MAIA, M. et al. The Use of the International Classification of Functioning, Disability and Health (ICF) Framework on Educational Planning: Promoting an Environmental Approach. International Journal of Cross-Disciplinary Subjects in Education, Special Issue, v. 2, n.1, (in press).

SIMEONSSON, R. J. Defining and Classifying Disability in Children. In: WORKSHOP ON DISABILITY IN AMERICA A NEW LOOK, 2005, Washington D. C. Summary and Background Papers...Washington D. C.: The National Academies Press, 2006. 276. p. 67-87.

SIMEONSSON, R. J.; LOLLAR, D. J. Individual with Disabilities Education Act. (IDEA) In:

ALBRECHT, G. (Eds). Encyclopedia of Disability. Thousand Oaks, CA: Sage Publications, 2006. p. 945-947.

SKRTIC, T. M. The special education paradox: equity as a way to excellence. In: HEHIR, T.; LATUS, T. (Eds.). Special Education at the Century's End. Cambridge: Harvard Educational Review, 1992. p. 203-272.

STAINBACK, S.; STAINBACK, W.; AYRES, B. Schools as inclusive communities. In: STAINBACK, W.; STAINBACK, S. (Eds.). Controversial Issues Confronting Special Education: Divergent Perspectives. Boston: Allyn \& Bacon, 1996. p. 31-43.

STERN, W. The Psychological Methods of Intelligence Testing. Baltimore: Warwick \& York, 1914.

VERBRUGGE, L.; JETTE, A. The disablement process. Social Science and Medicine, v. 38, n. 1, p. $1-14,1994$. 
WHITENECK, G.; FOUGEYROLLAS, P.; GERHART, K. A. Elaborating the model of disablement. In: FUHRER, M. J. (Ed.). Assessing medical rehabilitation practices: the promise of outcomes research. Baltimore: Paul H. Brookes Publishing Co., 1997. p. 91-102.

WHITENECK, G. Conceptual Models of Disability: Past, Present, and Future. In: WORKSHOP ON DISABILITY IN AMERICA A NEW LOOK, 2005, Washington D. C. Summary and Background Papers...Washington D. C.: The National Academies Press, 2006. 276. p. 50-66.

WHITTINGTON, J.; HOLLAND, A.; \& WEBB, T. Relationship between the IQ of people with Prader-Willi syndrome and that of their siblings: evidence for imprinted gene effects. Journal of Intellectual Disability Research, v. 53, p. 411-418, 2009.

ZAZZO, R. Le Devenir de l'intelligence. Paris: Presses Universitaires, 1946a. . Intelligence et quotient d'âges. Paris: Le Chemin des philosophes, 1946b

Recebido em: 26/11/2012

Aprovado em: 0312/2012 